\title{
Effects of platelet-rich plasma injection on pain, range of motion, and disability in adhesive capsulitis: A prospective, randomized-controlled study
}

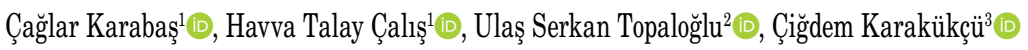 \\ ${ }^{1}$ Department of Physical Medicine and Rehabilitation, Kayseri City Hospital, Kayseri, Turkey \\ 2Department of Internal Medicine, Kayseri City Hospital, Kayseri, Turkey \\ ${ }^{3}$ Department of Biochemistry, Kayseri City Hospital, Kayseri, Turkey
}

Received: May 28, 2020 Accepted: December 04, 2020 Published online: December 01, 2021

\begin{abstract}
Objectives: In this study, we aimed to investigate the effectiveness of intra-articular platelet-rich plasma (PRP) injection in adhesive capsulitis.

Patients and methods: Between January 2019 and December 2019, a total of 40 patients (21 males, 19 females; mean age: $57.1 \pm 6.5$ years; range, 44 to 72 years) with idiopathic adhesive capsulitis were included. The patients were randomly assigned into two equal groups as the PRP and the control group. The PRP group received two doses of PRP via intra-articular route biweekly under ultrasound guidance. No injection was performed to the control group. In both groups, stretching and Codman exercises were applied as a homebased program. The Visual Analog Scale (VAS), range of motion (ROM), and Shoulder Pain and Disability Index (SPADI) scores were evaluated before the treatment and at 2, 6 and 12 weeks after the treatment.

Results: There were significant differences in all VAS, SPADI, and ROM scores at all time points after treatment compared to baseline in both groups. At the end of the study, there were significant differences in the active flexion, passive flexion, active abduction, passive abduction, and active external rotation scores at 12 weeks between the groups $(p=0.012, p=0.015, p=0.008, p=0.019$, and $p=0.040$, respectively). No significant difference was observed between the groups in terms of VAS and SPADI scores and the other parameters (active and passive extension, active and passive internal rotation, passive external rotation) at 2, 6, and 12 weeks ( $\mathrm{p}>0.05$ ).

Conclusion: The addition of PRP to exercise treatment can improve patients' joint mobility, but not pain and disability in patients with adhesive capsulitis.

Keywords: Adhesive capsulitis, disability, pain, platelet-rich plasma, range of motion.
\end{abstract}

Adhesive capsulitis, also known as frozen shoulder, defines a pathological process characterized by adhesion or excessive scarring in the glenohumeral joint, which causes stiffness, pain, and dysfunction in the shoulder. ${ }^{[1,2]}$ Adhesive capsulitis, which is considered that a large number of growth factors within the plasma content would increase tendon and cartilage tissue regeneration, is seen in the general population at a rate of approximately 3 to $5 \% \cdot{ }^{[3]}$ The clinical picture indicates a tendency to spontaneous recovery within one to three years. However, it demonstrates a resistant process in 20 to $40 \%$ of cases. $^{[2,3]}$

Platelet-rich plasma (PRP) obtained by centrifugation of whole blood is the plasma component that contains a higher platelet concentration than whole blood. ${ }^{[4]}$ Histopathological evidence shows that

Corresponding author: Ulaş Serkan Topaloğlu, MD. Kayseri Şehir Hastanesi Ị̧̇ Hastalıkları Kliniğ̈i, 38080 Kocasinan, Kayseri, Türkiye.

$$
\text { e-mail: ustop38@gmail.com }
$$


failure of repair mechanisms plays a role in the pathological process rather than inflammation in chronic soft tissue degeneration. ${ }^{[5]}$ The fact that PRP contains many growth factors has led to the use of PRP injections in the treatment of several musculoskeletal system disorders. ${ }^{[6,7]}$ It has been suggested that growth factors considered to play a role in the healing process can be potentially used in the treatment by enhancing their effects on tendon and cartilage tissue via local administration rather than lesions. ${ }^{[5]}$ Although there are increasing numbers of clinical trials investigating PRP injections in musculoskeletal system disorders in the literature, the effectiveness results are controversial. ${ }^{[8-11]}$ Similarly, for shoulder joints, numerous references are available regarding rotator cuff tendinopathy, bicipital tendinopathy, and the usage of PRP in superior labrum anterior-posterior lesions. ${ }^{[12-14]}$

Despite many studies on PRP treatment in various joint diseases, there are scarce comparative data on the use of PRP in adhesive capsulitis. In the present study, we aimed to investigate the effectiveness of PRP therapy in patients with adhesive capsulitis.

\section{PATIENTS AND METHODS}

This single-center, single-blind, parallel-group, prospective randomized-controlled, study was conducted at Physical Therapy and Rehabilitation outpatient clinic of Kayseri City Hospital between January 2019 and December 2019. A total of 61 patients aged between 18 and 75 years with shoulder pain for at least three months, 50\% limitation in at least one direction of range of motion (ROM), a Visual Analog Scale (VAS) score of $>5$, who underwent physical examination by a single physiatrist and were diagnosed with idiopathic adhesive capsulitis were screened. The patients were randomly assigned into two groups (PRP and the control) by another physiatrist, using the sealed envelope method at the beginning of the application. Patients who received an injection within the prior three months in the relevant region, those who received physical therapy in the relevant region, those with local infection, those with systemic infection or inflammatory disease (rheumatoid arthritis, hepatitis), those with diabetes mellitus, those with a history of malignancy (hematological or non-hematological), pregnant women, and those on systemic steroid therapy were excluded. Also, those with a full-thickness rotator cuff tear and patients having previous shoulder surgery were excluded from the study. Only those with partial rotator cuff tear together with adhesive capsulitis and patients with bursitis and tendinopathy were included in the study. A total of 18 patients were excluded from the study, as they had adhesive

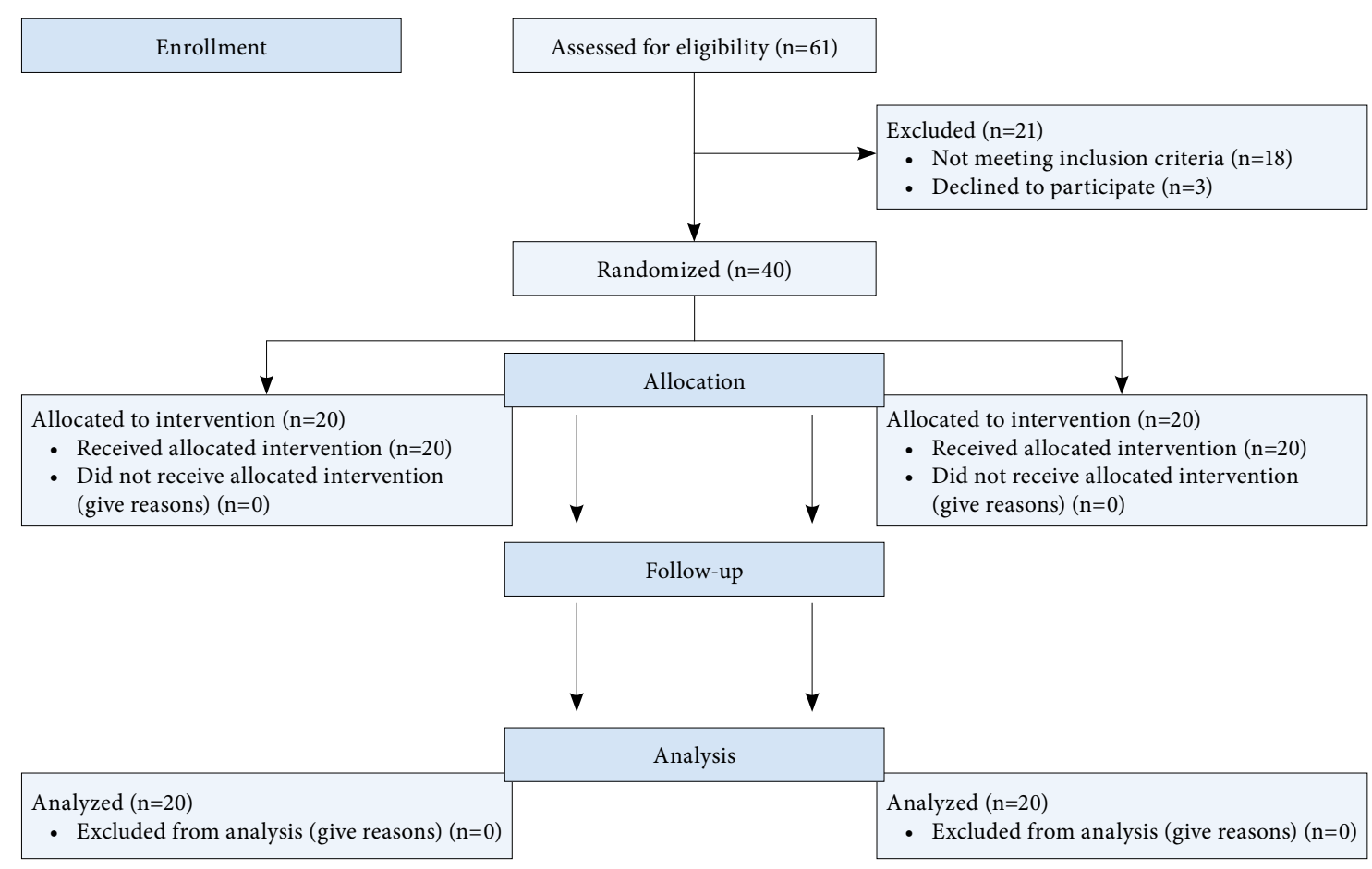

Figure 1. Study flow chart. 
capsulitis due to secondary etiology. Three patients left the study due to the transportation problem. Finally, a total of 40 patients ( 21 males, 19 females; mean age: $57.1 \pm 6.5$ years; range, 44 to 72 years) were included in the study. The study flow chart is shown in Figure 1.

A written informed consent was obtained from each patient. This study was approved by the Local Ethics Committee of Erciyes University Faculty of Medicine (No: 2017/272). The study was conducted in accordance with the principles of the Declaration of Helsinki.

Using sonography guidance, two doses of PRP $(3 \mathrm{~mL})$ were injected biweekly into the glenohumeral joint via a posterior approach by a physiatrist blinded to the history and physical examination findings in 20 patients in the PRP group. No PRP injection was performed to the control one. Besides, a home-based exercise program including proprioceptive neuromuscular facilitation stretching and Codman pendulum exercises ( $3 \times 10$ repeats) was prescribed to all patients in the study. Moreover, the patients were asked not to use non-steroidal anti-inflammatory drugs until the end of the study, whereas paracetamol was allowed as an analgesic.

\section{Preparation of platelet-rich plasma}

Manual preparation techniques were used in the study. ${ }^{[15]}$ In each patient, $24 \mathrm{~mL}$ of venous blood samples $(8 \mathrm{~mL} \times 3)$ were drawn into three sterile tubes containing $2 \mathrm{~mL}$ of anticoagulant citrate dextrose solution, from the cubital veins. The tubes were, then, centrifuged at $1,195 \mathrm{rpm}$ for $20 \mathrm{~min}$, resulting in three layers of whole blood sample: the first layer, plasma (superior layer); the second layer, buffy coat (platelets plus leukocytes); and the third layer, erythrocytes (inferior layer). The first and second layers were transferred to three empty tubes and re-centrifuged at $1,890 \mathrm{rpm}$ for $15 \mathrm{~min}$ under laminar flow. This resulted in two layers. The first layer, the platelet poor superior layer was collected by syringe but was not used. The second layer was the platelet-rich inferior layer ( $1.5 \mathrm{~mL}$ per tube). From three tubes, $4.5 \mathrm{~mL}$ of PRP was obtained and divided into three tubes. Two tubes $(3 \mathrm{~mL})$ were used for treatment, while one tube $(1.5 \mathrm{~mL})$ was assigned for a platelet count to ensure the desired platelet count.

\section{Ultrasound-guided intra-articular platelet-rich plasma injection technique}

In the study, the glenohumeral joint was identified between the infraspinatus muscle and humeral head via a posterior approach by a Philips ClearVue 550 device (Philips Ultrasound Inc, Pennsylvania, USA), using a $512 \mathrm{~Hz}$ linear probe. Under appropriate sterile conditions, the intra-articular space was punctured by a 21-G needle. After confirmation of the needle tip within the intra-articular space, $3 \mathrm{~mL}$ of autologous PRP was administered into the capsule and the procedure was completed. The procedure was repeated two weeks after the first injection by the same physiatrist using the same technique. The injection procedure, randomization, and questionnaire were performed by different physiatrists.

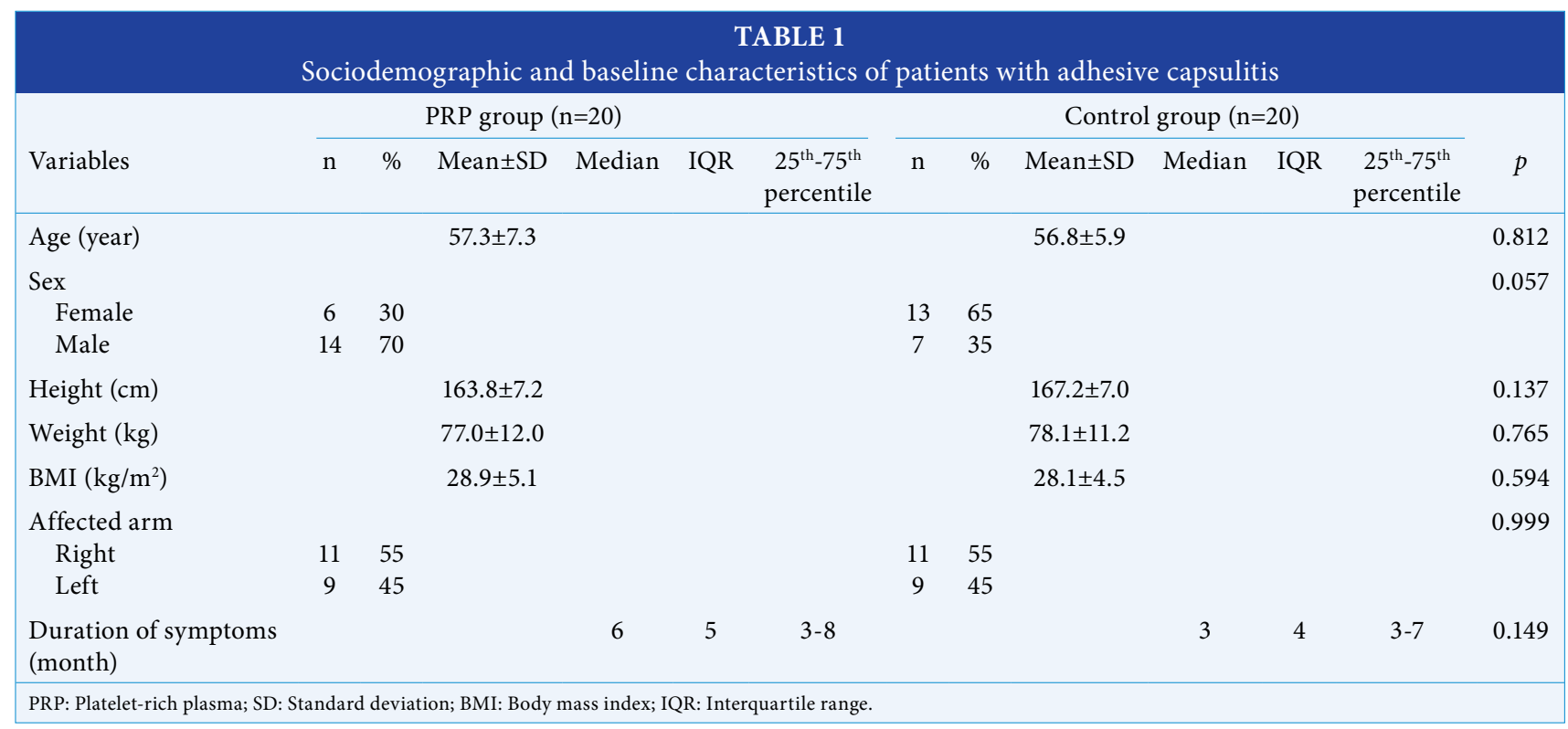




\section{Outcome measures}

Demographic characteristics of the patients were recorded. The pain was assessed by 10 -points for VAS ( 0 , no pain; 10 , worst pain ever). In all patients, the pain was assessed separately as daytime pain (VAS-daytime), night pain (VAS-night), and during movement (VAS-movement).

In all patients, the Shoulder Pain and Disability Index (SPADI) was used to assess shoulder pain and disability by the same physiatrists. Pain and disability subscales were calculated as a mean value of items rated with a 0-100 scale, the highest scores indicating the most severe pain and disability. The SPADI, which is a scale used in adhesive capsulitis studies, is also a valid and reliable questionnaire in the Turkish population. ${ }^{[16,17]}$

The ROM was separately assessed as active and passive ROM (flexion, abduction, internal rotation, external rotation, extension).

\begin{tabular}{|c|c|c|c|}
\hline \multicolumn{4}{|c|}{$\begin{array}{c}\text { TABLE } 2 \\
\text { Non-parametric analysis of longitudinal data in factorial } \\
\text { experiments for the VAS and SPADI scores }\end{array}$} \\
\hline Source of variation & Wald & $\mathrm{df}$ & $p$ \\
\hline \multicolumn{4}{|l|}{ VAS pain score (day) } \\
\hline Treatment & 1.693 & 1 & 0.193 \\
\hline Time (week) & 212.409 & 3 & $<0.001$ \\
\hline Treatment $\mathrm{x}$ time (week) & 3.676 & 3 & 0.299 \\
\hline \multicolumn{4}{|l|}{ VAS pain score (night) } \\
\hline Treatment & 1.525 & 1 & 0.217 \\
\hline Time (week) & 142.226 & 3 & $<0.001$ \\
\hline Treatment $\mathrm{x}$ time (week) & 1.357 & 3 & 0.716 \\
\hline \multicolumn{4}{|l|}{ VAS pain score (motion) } \\
\hline Treatment & 0.001 & 1 & 0.985 \\
\hline Time (week) & 185.157 & 3 & $<0.001$ \\
\hline Treatment x time (week) & 2.434 & 3 & 0.487 \\
\hline \multicolumn{4}{|l|}{ SPADI score (pain) } \\
\hline Treatment & 0.014 & 1 & 0.906 \\
\hline Time (week) & 183.181 & 3 & $<0.001$ \\
\hline Treatment $\mathrm{x}$ time (week) & 2.606 & 3 & 0.456 \\
\hline \multicolumn{4}{|l|}{ SPADI score (disability) } \\
\hline Treatment & 0.011 & 1 & 0.915 \\
\hline Time (week) & 231.528 & 3 & $<0.001$ \\
\hline Treatment $\mathrm{x}$ time (week) & 2.492 & 3 & 0.477 \\
\hline \multicolumn{4}{|l|}{ SPADI score (total) } \\
\hline Treatment & 0.021 & 1 & 0.884 \\
\hline Time (week) & 239.002 & 3 & $<0.001$ \\
\hline Treatment $\mathrm{x}$ time (week) & 3.490 & 3 & 0.322 \\
\hline
\end{tabular}

The VAS-daytime, VAS-night, VAS-movement, active and passive ROM, SPADI-pain, SPADI disability, and SPADI-total were assessed at baseline and at 2,6 , and 12 weeks.

\begin{tabular}{|c|c|c|c|}
\hline \multicolumn{4}{|c|}{$\begin{array}{c}\text { TABLE } 3 \\
\text { Non-parametric analysis of longitudinal data in factorial } \\
\text { experiments for the ROM scores }\end{array}$} \\
\hline Source of variation & Wald & $\mathrm{df}$ & $p$ \\
\hline \multicolumn{4}{|l|}{ Flexion (active) } \\
\hline Treatment & 1.810 & 1 & 0.179 \\
\hline Time (week) & 115.875 & 3 & $<0.001$ \\
\hline Treatment $\mathrm{x}$ time (week) & 5.612 & 3 & 0.132 \\
\hline \multicolumn{4}{|l|}{ Flexion (passive) } \\
\hline Treatment & 2.504 & 1 & 0.114 \\
\hline Time (week) & 135.437 & 3 & $<0.001$ \\
\hline Treatment $\mathrm{x}$ time (week) & 5.041 & 3 & 0.169 \\
\hline \multicolumn{4}{|l|}{ Extension (active) } \\
\hline Treatment & 0.109 & 1 & 0.741 \\
\hline Time (week) & 84.240 & 3 & $<0.001$ \\
\hline Treatment $\mathrm{x}$ time (week) & 3.208 & 3 & 0.361 \\
\hline \multicolumn{4}{|l|}{ Extension (passive) } \\
\hline Treatment & 0.094 & 1 & 0.759 \\
\hline Time (week) & 140.782 & 3 & $<0.001$ \\
\hline Treatment $\mathrm{x}$ time (week) & 2.126 & 3 & 0.547 \\
\hline \multicolumn{4}{|l|}{ Abduction (active) } \\
\hline Treatment & 0.716 & 1 & 0.398 \\
\hline Time (week) & 225.192 & 3 & $<0.001$ \\
\hline Treatment $\mathrm{x}$ time (week) & 11.908 & 3 & 0.008 \\
\hline \multicolumn{4}{|l|}{ Abduction (passive) } \\
\hline Treatment & 0.939 & 1 & 0.333 \\
\hline Time (week) & 231.315 & 3 & $<0.001$ \\
\hline Treatment $\mathrm{x}$ time (week) & 11.285 & 3 & 0.010 \\
\hline \multicolumn{4}{|l|}{ Internal rotation (active) } \\
\hline Treatment & 0.037 & 1 & 0.846 \\
\hline Time (week) & 163.217 & 3 & $<0.001$ \\
\hline Treatment $\mathrm{x}$ time (week) & 3.730 & 3 & 0.292 \\
\hline \multicolumn{4}{|l|}{ Internal rotation (passive) } \\
\hline Treatment & 0.012 & 1 & 0.913 \\
\hline Time (week) & 142.250 & 3 & $<0.001$ \\
\hline Treatment $\mathrm{x}$ time (week) & 4.822 & 3 & 0.185 \\
\hline \multicolumn{4}{|l|}{ External rotation (active) } \\
\hline Treatment & 0.595 & 1 & 0.440 \\
\hline Time (week) & 135.028 & 3 & $<0.001$ \\
\hline Treatment $\mathrm{x}$ time (week) & 7.892 & 3 & 0.048 \\
\hline \multicolumn{4}{|l|}{ External rotation (passive) } \\
\hline Treatment & 0.393 & 1 & 0.531 \\
\hline Time (week) & 157.299 & 3 & $<0.001$ \\
\hline Treatment $\mathrm{x}$ time (week) & 7.247 & 3 & 0.064 \\
\hline
\end{tabular}




\section{Statistical analysis}

Study power analysis was performed using the $\mathrm{G}^{\star}$ Power version 3.0.10 software (Heinrich-Heine Universität Düsseldorf, Düsseldorf, Germany). The post-hoc power analysis was calculated based on the Cohen's d and partial eta-square effect size statistics. All these calculations are conducted using $\mathrm{R}$ 3.6.1 (www.r-project.org) statistical software.

Statistical analysis was performed using the TURCOSA (Turcosa Analytics Ltd. Co., www. turcosa.com.tr) and nparLD library of $\mathrm{R}$ 3.6.1 (www.r-project.org) software. ${ }^{[18]}$ Histogram, q-q plots, and Shapiro-Wilk's tests were used to assess the data normality. The Levene test was used to test variance homogeneity. Normally distributed quantitative data were expressed in mean \pm standard deviation (SD), while non-normally distributed data were expressed in median and interquartile range (IQR). Qualitative data were expressed in number and frequency. Pearson chi-square analysis was used for comparison of categorical variables. To identify the main and interaction effects of treatment and time points on VAS, SPADI and ROM scores, non-parametric analysis of longitudinal data in factorial experiments was carried out. ${ }^{[19]}$ Wald-type statistics were calculated for testing treatment group and time effects, and interaction. Experimental results were given in Wald statistic, degrees of freedom and $p$ values. Inter-group comparisons were performed using a two-sided independent samples t-test and Mann-Whitney $\mathrm{U}$ test. Intra-group comparisons were performed using one-way repeated measures analysis of variance (ANOVA) and Friedman tests. Bonferroni and Nemenyi tests were used simultaneously for multiple comparisons. A $p$ value of $<0.05$ was considered statistically significant.

TABLE 4

Changes from baseline in the VAS scores between PRP and control groups

\begin{tabular}{|c|c|c|c|c|c|c|c|c|}
\hline \multirow[b]{2}{*}{ VAS pain score } & \multicolumn{3}{|c|}{ PRP group $(n=20)$} & \multicolumn{3}{|c|}{ Control group $(n=20)$} & \multirow[b]{2}{*}{$p^{*}$} & \multirow[b]{2}{*}{ Post-power } \\
\hline & Median & IQR & $25^{\text {th }}-75^{\text {th }}$ quartiles & Median & IQR & $25^{\text {th }}-75^{\text {th }}$ quartiles & & \\
\hline \multicolumn{9}{|l|}{ Day } \\
\hline Baseline & $8.0^{\mathrm{a}}$ & 5 & $5.3 / 10.0$ & $7.0^{\mathrm{a}}$ & 4 & $4.0 / 8.0$ & 0.121 & 0.333 \\
\hline 2 weeks & $5.0^{\mathrm{ab}}$ & 5 & $3.3 / 7.8$ & $4.0^{\mathrm{b}}$ & 4 & $2.0 / 6.0$ & 0.091 & 0.416 \\
\hline 6 weeks & $3.5^{\mathrm{bc}}$ & 3 & $2.3 / 5.0$ & $2.0^{\mathrm{bc}}$ & 3 & $1.0 / 4.0$ & 0.242 & 0.138 \\
\hline 12 weeks & $2.0^{c}$ & 2 & $1.0 / 3.0$ & $2.0^{c}$ & 3 & $1.0 / 3.8$ & 0.883 & 0.059 \\
\hline Difference (2 weeks-Baseline) & -2.0 & 2 & $-3.0 /-1.0$ & -2.0 & 1 & $-3.0 /-2.0$ & 0.461 & 0.087 \\
\hline Difference (6 weeks-Baseline) & -4.5 & 3 & $-5.0 /-3.0$ & -3.0 & 3 & $-5.0 /-2.0$ & 0.355 & 0.104 \\
\hline Difference (12 weeks-Baseline) & -5.0 & & $-7.0 /-4.0$ & -5.0 & 4 & $-5.8 /-2.0$ & 0.134 & 0.228 \\
\hline$p^{* *}$ & \multicolumn{3}{|c|}{$<0.001$} & \multicolumn{3}{|c|}{$<0.001$} & & \\
\hline Post-power & \multicolumn{3}{|c|}{1.000} & \multicolumn{3}{|c|}{1.000} & & \\
\hline \multicolumn{9}{|l|}{ Night } \\
\hline Baseline & $9.0^{\mathrm{a}}$ & 1 & $9.0 / 10.0$ & $8.5^{\mathrm{a}}$ & 2 & $8.0 / 9.8$ & 0.102 & 0.270 \\
\hline 2 weeks & $7.0^{\mathrm{b}}$ & 5 & $4.0 / 8.8$ & $5.0^{\mathrm{ab}}$ & 4 & $3.0 / 7.0$ & 0.134 & 0.341 \\
\hline 6 weeks & $4.0^{\mathrm{bc}}$ & 3 & $2.0 / 5.0$ & $3.0^{\mathrm{bc}}$ & 4 & $1.3 / 5.5$ & 0.445 & 0.083 \\
\hline 12 weeks & $2.5^{\mathrm{c}}$ & 5 & $1.0 / 5.8$ & $2.0^{c}$ & 4 & $1.0 / 5.0$ & 0.718 & 0.078 \\
\hline Difference (2 weeks-Baseline) & -2.0 & 3 & $-3.8 /-1.0$ & -2.0 & 4 & $-4.8 /-1.3$ & 0.547 & 0.107 \\
\hline Difference (6 weeks-Baseline) & -5.0 & 4 & $-7.0 /-3.0$ & -5.0 & 5 & $6.8 /-2.3$ & 0.841 & 0.064 \\
\hline Difference (12 weeks-Baseline) & -7.0 & 5 & $-8.0 /-3.0$ & -5.5 & 5 & $-7.8 /-3.3$ & 0.620 & 0.061 \\
\hline$p^{* *}$ & \multicolumn{3}{|c|}{$<0.001$} & \multicolumn{3}{|c|}{$<0.001$} & & \\
\hline Post-power & \multicolumn{3}{|c|}{1.000} & \multicolumn{3}{|c|}{1.000} & & \\
\hline \multicolumn{9}{|l|}{ Motion } \\
\hline Baseline & $10.0^{\mathrm{a}}$ & 2 & $8.0 / 10.0$ & $9.0^{\mathrm{a}}$ & 2 & $8.0 / 10.0$ & 0.165 & 0.207 \\
\hline 2 weeks & $6.0^{\mathrm{ab}}$ & 3 & $5.0 / 8.0$ & $6.0^{\mathrm{b}}$ & 3 & $5.0 / 7.8$ & 0.738 & 0.068 \\
\hline 6 weeks & $4.0^{\mathrm{bc}}$ & 3 & $2.3 / 5.0$ & $4.5^{\mathrm{bc}}$ & 5 & $2.3 / 7.0$ & 0.602 & 0.095 \\
\hline 12 weeks & $2.0^{c}$ & 5 & $0.3 / 5.0$ & $2.0^{c}$ & 3 & $2.0 / 5.0$ & 0.398 & 0.104 \\
\hline Difference (2 weeks-Baseline) & -5.0 & 3 & $-2.0 /-1.3$ & -3.0 & 3 & $-2.0 /-1.3$ & 0.583 & 0.113 \\
\hline Difference (6 weeks-Baseline) & -5.0 & 3 & $-6.8 /-4.0$ & -3.5 & 4 & $-6.0 /-2.0$ & 0.149 & 0.300 \\
\hline Difference (12 weeks-Baseline) & -6.0 & 5 & $-9.0 /-4.3$ & -5.0 & 4 & $-7.0 /-3.3$ & 0.149 & 0.304 \\
\hline$p^{* *}$ & \multicolumn{3}{|c|}{$<0.001$} & \multicolumn{3}{|c|}{$<0.001$} & & \\
\hline Post-power & \multicolumn{3}{|c|}{1.000} & \multicolumn{3}{|c|}{1.000} & & \\
\hline
\end{tabular}

VAS: Visual Analog Scale; PRP: platelet-rich plasma; IQR: Interquartile range; ${ }^{*}$ p: Between group comparison using the Mann-Whitney U test; ${ }^{* *}$ : Within group comparison using Friedman test followed by Nemenyi multiple comparison test. Significant $p$ values are shown in bold. Different superscripts in the same column indicate a statistically significant difference between time points. 


\section{RESULTS}

Baseline characteristics of the PRP and control groups are summarized in Table 1. There were no significant differences in the age, sex, height, weight, body mass index, and involved arm between the groups $(\mathrm{p}>0.05)$. The median duration of symptoms was six (range, 3 to 8 ) years in the PRP group and three (range, 3 to 7 ) years in the control group, indicating no significant differences between the groups $(\mathrm{p}=0.812$ and $\mathrm{p}=0.149$, respectively).

However, there were significant differences in all status of VAS and SPADI scores for all time points after treatment compared to baseline in both groups $(\mathrm{p}<0.05)$. At the end of the study, there were no significant differences in all of VAS and SPADI scores between the PRP group and control group ( $p>0.05)$. In addition, there were significant differences in all status of active and passive ROM compared to baseline in both groups. A significant difference was observed in the active flexion, passive flexion, active abduction, passive abduction, and active external rotation scores at 12 weeks between the groups $(p=0.012, p=0.015, p=0.008, p=0.019$, and $\mathrm{p}=0.040$, respectively), while no significant difference was noted between the groups in the other parameters (active and passive extension, active and passive internal rotation, passive external rotation) at 12 weeks $(\mathrm{p}=0.156, \mathrm{p}=0.167, \mathrm{p}=0.218, \mathrm{p}=0.110$, and $\mathrm{p}=0.081$, respectively) (Table $2-10$ ).

TABLE 5

Changes from baseline in the SPADI scores between PRP and control groups

\begin{tabular}{|c|c|c|c|c|c|c|c|c|}
\hline & \multicolumn{3}{|c|}{ PRP group $(n=20)$} & \multicolumn{3}{|c|}{ Control group $(n=20)$} & \multirow[b]{2}{*}{$p^{*}$} & \multirow[b]{2}{*}{ Post-power } \\
\hline SPADI score & Median & IQR & $25^{\text {th }}-75^{\text {th }}$ quartiles & Median & IQR & $25^{\text {th }}-75^{\text {th }}$ quartiles & & \\
\hline \multicolumn{9}{|l|}{ Pain } \\
\hline Baseline & $92.0 \mathrm{a}$ & 17.5 & $78.5 / 96.0$ & $79.0^{\mathrm{a}}$ & 19.0 & $72.5 / 91.5$ & 0.121 & 0.128 \\
\hline 2 weeks & $68.0 \mathrm{a}$ & 38.5 & $40.5 / 79.0$ & $53.0^{\mathrm{ab}}$ & 37.5 & $40.5 / 78.0$ & 0.640 & 0.083 \\
\hline 6 weeks & $39.0 \mathrm{~b}$ & 33.0 & $20.5 / 53.5$ & $40.0^{\mathrm{bc}}$ & 25.5 & $26.0 / 51.5$ & 0.841 & 0.101 \\
\hline 12 weeks & $21.0 \mathrm{~b}$ & 20.0 & $13.0 / 33.0$ & $21.0^{c}$ & 23.8 & $12.0 / 35.7$ & 0.968 & 0.050 \\
\hline Difference (2 weeks-Baseline) & -23.0 & 19 & $-31.0 /-12.0$ & -20.0 & 23 & $-34.0 /-10.5$ & 0.968 & 0.051 \\
\hline Difference (6 weeks-Baseline) & -47.0 & 21 & $-56.0 /-34.8$ & -40.0 & 36 & $-50.0 /-26.0$ & 0.242 & 0.252 \\
\hline Difference (12 weeks-Baseline) & -62.0 & 41 & $-76.0 /-35.3$ & -57.0 & 25 & $-67.5 /-40.8$ & 0.495 & 0.079 \\
\hline$p^{\star *}$ & \multicolumn{3}{|c|}{$<0.001$} & \multicolumn{3}{|c|}{$<0.001$} & & \\
\hline Post-power & \multicolumn{3}{|c|}{1.000} & \multicolumn{3}{|c|}{1.000} & & \\
\hline \multicolumn{9}{|l|}{ Disability } \\
\hline Baseline & $80.0^{\mathrm{a}}$ & 26.9 & $71.5 / 88.4$ & $72.5^{\mathrm{a}}$ & 27.6 & $58.6 / 86.2$ & 0.165 & 0.194 \\
\hline 2 weeks & $50.6^{\mathrm{b}}$ & 39.9 & $33.9 / 73.8$ & $47.5^{\mathrm{b}}$ & 34.9 & $34.6 / 69.0$ & 0.862 & 0.053 \\
\hline 6 weeks & $35.0^{\mathrm{bc}}$ & 22.3 & $16.2 / 38.5$ & $33.1^{\mathrm{bc}}$ & 32.8 & $19.0 / 51.8$ & 0.602 & 0.109 \\
\hline 12 weeks & $15.6^{c}$ & 28.8 & $0.0 / 28.7$ & $18.7^{c}$ & 22.8 & $9.0 / 31.8$ & 0.341 & 0.078 \\
\hline Difference (2 weeks-Baseline) & -23.0 & 19 & $-31.0 /-12.0$ & -23.0 & 19 & $-31.0 /-12.0$ & 0.495 & 0.120 \\
\hline Difference (6 weeks-Baseline) & -23.0 & 19 & $-31.0 /-12.0$ & -23.0 & 19 & $-31.0 /-12.0$ & 0.157 & 0.392 \\
\hline Difference (12 weeks-Baseline) & -23.0 & 19 & $-31.0 /-12.0$ & -23.0 & 19 & $-31.0 /-12.0$ & 0.149 & 0.245 \\
\hline$p^{* *}$ & \multicolumn{3}{|c|}{$<0.001$} & \multicolumn{3}{|c|}{$<0.001$} & & \\
\hline Post-power & \multicolumn{3}{|c|}{1.000} & \multicolumn{3}{|c|}{1.000} & & \\
\hline \multicolumn{9}{|l|}{ Total } \\
\hline Baseline & $86.2^{\mathrm{a}}$ & 15.2 & $78.6 / 93.8^{\mathrm{a}}$ & $78.5^{\mathrm{a}}$ & 20.9 & $69.0 / 90.0$ & 0.121 & 0.137 \\
\hline 2 weeks & $60.4^{\mathrm{a}}$ & 38.6 & $39.0 / 77.6^{\mathrm{a}}$ & $55.0^{\mathrm{b}}$ & 30.9 & $40.1 / 71.1$ & 0.758 & 0.053 \\
\hline 6 weeks & $36.9^{\mathrm{b}}$ & 30.2 & $17.3 / 47.4^{\mathrm{b}}$ & $35.4^{\mathrm{bc}}$ & 30.8 & $24.1 / 54.9$ & 0.620 & 0.146 \\
\hline 12 weeks & $17.3^{\mathrm{b}}$ & 30.0 & $7.1 / 37.1^{\mathrm{b}}$ & $20.0^{c}$ & 23.8 & $10.1 / 34.0$ & 0.620 & 0.061 \\
\hline Difference (2 weeks-Baseline) & -23.0 & 19 & $-31.0 /-12.0$ & -23.0 & 19 & $-31.0 /-12.0$ & 0.583 & 0.087 \\
\hline Difference (6 weeks-Baseline) & -23.0 & 19 & $-31.0 /-12.0$ & -23.0 & 19 & $-31.0 /-12.0$ & 0.183 & 0.373 \\
\hline Difference (12 weeks-Baseline) & -23.0 & 19 & $-31.0 /-12.0$ & -23.0 & 19 & $-31.0 /-12.0$ & 0.142 & 0.137 \\
\hline$p^{\star *}$ & \multicolumn{3}{|c|}{$<0.001$} & \multicolumn{3}{|c|}{$<0.001$} & & \\
\hline Post-power & \multicolumn{3}{|c|}{1.000} & \multicolumn{3}{|c|}{1.000} & & \\
\hline
\end{tabular}




\begin{tabular}{|c|c|c|c|c|}
\hline \multicolumn{5}{|c|}{$\begin{array}{c}\text { TABLE } 6 \\
\text { Changes from baseline in the flexion scores between PRP and control groups }\end{array}$} \\
\hline \multirow[b]{2}{*}{ Flexion } & PRP group $(n=20)$ & Control group $(n=20)$ & \multirow[b]{2}{*}{$p^{*}$} & \multirow[b]{2}{*}{ Post-power } \\
\hline & Mean \pm SD & Mean \pm SD & & \\
\hline \multicolumn{5}{|l|}{ Active } \\
\hline Baseline & $94.0 \pm 28.5^{\mathrm{a}}$ & $103.2 \pm 14.9^{\mathrm{a}}$ & 0.208 & 0.239 \\
\hline 2 weeks & $126.0 \pm 31.3^{\mathrm{b}}$ & $119.2 \pm 16.8^{\mathrm{ab}}$ & 0.368 & 0.144 \\
\hline 6 weeks & $135.0 \pm 26.7^{\mathrm{bc}}$ & $124.2 \pm 22.0^{\mathrm{bc}}$ & 0.165 & 0.282 \\
\hline 12 weeks & $154.0 \pm 25.0^{c}$ & $134.0 \pm 23.0^{c}$ & 0.012 & 0.725 \\
\hline Difference (2 weeks-Baseline) & $32.5 \pm 28.8$ & $16.0 \pm 16.1$ & 0.031 & 0.589 \\
\hline Difference (6 weeks-Baseline) & $41.3 \pm 26.5$ & $21.0 \pm 20.2$ & 0.010 & 0.756 \\
\hline Difference (12 weeks-Baseline) & $60.0 \pm 37.8$ & $30.8 \pm 23.2$ & 0.005 & 0.820 \\
\hline$p^{* *}$ & $<0.001$ & $<0.001$ & & \\
\hline Post-power & 1.000 & 1.000 & & \\
\hline \multicolumn{5}{|l|}{ Passive } \\
\hline Baseline & $106.2 \pm 27.6^{\mathrm{a}}$ & $109.7 \pm 17.0^{\mathrm{a}}$ & 0.633 & 0.076 \\
\hline 2 weeks & $134.5 \pm 32.6^{\mathrm{ab}}$ & $127.0 \pm 18.5^{\mathrm{ab}}$ & 0.378 & 0.140 \\
\hline 6 weeks & $145.0 \pm 24.0^{\mathrm{bc}}$ & $132.0 \pm 21.9^{\mathrm{bc}}$ & 0.082 & 0.413 \\
\hline 12 weeks & $161.5 \pm 22.7^{c}$ & $142.7 \pm 23.7^{c}$ & 0.015 & 0.701 \\
\hline Difference (2 weeks-Baseline) & $28.3 \pm 20.5$ & $17.3 \pm 16.9$ & 0.072 & 0.438 \\
\hline Difference (6 weeks-Baseline) & $38.8 \pm 17.2$ & $22.3 \pm 19.5$ & 0.007 & 0.789 \\
\hline Difference (12 weeks-Baseline) & $55.3 \pm 32.8$ & $33.0 \pm 24.1$ & 0.019 & 0.664 \\
\hline$p^{* *}$ & $<0.001$ & $<0.001$ & & \\
\hline Post-power & 1.000 & 1.000 & & \\
\hline
\end{tabular}

\begin{tabular}{|c|c|c|c|c|}
\hline \multicolumn{5}{|c|}{$\begin{array}{l}\text { TABLE } 7 \\
\text { Changes from baseline in the extension scores between PRP and control groups }\end{array}$} \\
\hline \multirow[b]{2}{*}{ Extension } & \multirow{2}{*}{$\begin{array}{c}\text { PRP group }(\mathrm{n}=20) \\
\text { Mean } \pm \text { SD }\end{array}$} & \multirow{2}{*}{$\frac{\text { Control group }(\mathrm{n}=20)}{\text { Mean } \pm \text { SD }}$} & \multirow[b]{2}{*}{$p^{*}$} & \multirow[b]{2}{*}{ Post-power } \\
\hline & & & & \\
\hline \multicolumn{5}{|l|}{ Active } \\
\hline Baseline & $42.5 \pm 14.1^{\mathrm{a}}$ & $43.7 \pm 9.0^{\mathrm{a}}$ & 0.741 & 0.062 \\
\hline 2 weeks & $51.0 \pm 11.5^{\mathrm{ab}}$ & $51.2 \pm 9.1^{\mathrm{ab}}$ & 0.940 & 0.051 \\
\hline 6 weeks & $54.2 \pm 7.4^{\mathrm{b}}$ & $52.7 \pm 10.0^{\mathrm{b}}$ & 0.596 & 0.082 \\
\hline 12 weeks & $58.2 \pm 4.9^{\mathrm{b}}$ & $55.0 \pm 8.7^{\mathrm{b}}$ & 0.156 & 0.292 \\
\hline Difference (2 weeks-Baseline) & $8.5 \pm 13.6$ & $7.5 \pm 8.5$ & 0.782 & 0.059 \\
\hline Difference (6 weeks-Baseline) & $11.8 \pm 13.0$ & $9.0 \pm 10.1$ & 0.459 & 0.113 \\
\hline Difference (12 weeks-Baseline) & $15.8 \pm 14.9$ & $11.3 \pm 10.1$ & 0.271 & 0.193 \\
\hline$p^{* *}$ & $<0.001$ & $<0.001$ & & \\
\hline Post-power & 1.000 & 1.000 & & \\
\hline \multicolumn{5}{|l|}{ Passive } \\
\hline Baseline & $45.5 \pm 8.2^{\mathrm{a}}$ & $46.5 \pm 10.0^{\mathrm{a}}$ & 0.732 & 0.063 \\
\hline 2 weeks & $54.0 \pm 9.8^{\mathrm{b}}$ & $54.5 \pm 7.5^{\mathrm{b}}$ & 0.858 & 0.054 \\
\hline 6 weeks & $56.7 \pm 4.9^{\mathrm{b}}$ & $55.0 \pm 7.7^{\mathrm{b}}$ & 0.401 & 0.132 \\
\hline 12 weeks & $59.5 \pm 1.5^{\mathrm{b}}$ & $57.25 \pm 6.9^{\mathrm{b}}$ & 0.167 & 0.279 \\
\hline Difference (2 weeks-Baseline) & $8.5 \pm 8.1$ & $8.0 \pm 9.2$ & 0.857 & 0.054 \\
\hline Difference (6 weeks-Baseline) & $11.3 \pm 7.0$ & $8.5 \pm 10.5$ & 0.338 & 0.157 \\
\hline Difference (12 weeks-Baseline) & $14.0 \pm 8.0$ & $10.8 \pm 10.2$ & 0.269 & 0.194 \\
\hline$p^{* *}$ & $<0.001$ & $<0.001$ & & \\
\hline Post-power & 1.000 & 1.000 & & \\
\hline
\end{tabular}




\begin{tabular}{|c|c|c|c|c|}
\hline \multicolumn{5}{|c|}{$\begin{array}{l}\text { TABLE } 8 \\
\text { Changes from baseline in the abduction scores between PRP and control groups }\end{array}$} \\
\hline \multirow[b]{2}{*}{ Abduction } & \multirow{2}{*}{$\frac{\text { PRP group }(\mathrm{n}=20)}{\text { Mean } \pm \text { SD }}$} & \multirow{2}{*}{$\frac{\text { Control group }(\mathrm{n}=20)}{\text { Mean } \pm \text { SD }}$} & \multirow[b]{2}{*}{$p^{*}$} & \multirow[b]{2}{*}{ Post-power } \\
\hline & & & & \\
\hline \multicolumn{5}{|l|}{ Active } \\
\hline Baseline & $77.7 \pm 20.6^{\mathrm{a}}$ & $87.0 \pm 15.5^{\mathrm{a}}$ & 0.118 & 0.345 \\
\hline 2 weeks & $114.0 \pm 35.6^{\mathrm{b}}$ & $112.2 \pm 21.1^{\mathrm{b}}$ & 0.851 & 0.054 \\
\hline 6 weeks & $123.2 \pm 32.0^{\mathrm{bc}}$ & $110.5 \pm 26.9^{b}$ & 0.182 & 0.263 \\
\hline 12 weeks & $152.7 \pm 29.7^{c}$ & $126.2 \pm 30.3^{\mathrm{b}}$ & 0.008 & 0.776 \\
\hline Difference (2 weeks-Baseline) & $36.3 \pm 23.4$ & $25.3 \pm 19.1$ & 0.112 & 0.354 \\
\hline Difference (6 weeks-Baseline) & $45.5 \pm 24.8$ & $23.5 \pm 25.1$ & 0.008 & 0.777 \\
\hline Difference (12 weeks-Baseline) & $75.0 \pm 28.5$ & $39.3 \pm 29.9$ & $<0.001$ & 0.965 \\
\hline$p^{* *}$ & $<0.001$ & $<0.001$ & & \\
\hline Post-power & 1.000 & 1.000 & & \\
\hline \multicolumn{5}{|l|}{ Passive } \\
\hline Baseline & $85.5 \pm 20.9^{\mathrm{a}}$ & $94.5 \pm 19.5^{\mathrm{a}}$ & 0.168 & 0.278 \\
\hline 2 weeks & $127.5 \pm 39.7^{\mathrm{b}}$ & $120.0 \pm 23.3^{\mathrm{b}}$ & 0.471 & 0.109 \\
\hline 6 weeks & $131.7 \pm 30.9^{\mathrm{b}}$ & $119.5 \pm 29.1^{\mathrm{b}}$ & 0.205 & 0.242 \\
\hline 12 weeks & $161.2 \pm 28.3^{\mathrm{b}}$ & $138.2 \pm 31.0^{\mathrm{b}}$ & 0.019 & 0.663 \\
\hline Difference (2 weeks-Baseline) & $42.0 \pm 29.3$ & $25.5 \pm 17.9$ & 0.039 & 0.553 \\
\hline Difference (6 weeks-Baseline) & $46.3 \pm 26.3$ & $25.0 \pm 23.8$ & 0.011 & 0.743 \\
\hline Difference (12 weeks-Baseline) & $75.8 \pm 30.8$ & $43.8 \pm 28.4$ & 0.002 & 0.915 \\
\hline$p^{\star *}$ & $<0.001$ & $<0.001$ & & \\
\hline Post-power & 1.000 & 1.000 & & \\
\hline
\end{tabular}

\begin{tabular}{|c|c|c|c|c|}
\hline \multicolumn{5}{|c|}{$\begin{array}{r}\text { TABLE } 9 \\
\text { Changes from baseline in the internal rotation } \mathrm{s}\end{array}$} \\
\hline \multirow[b]{2}{*}{ Internal rotation } & \multirow{2}{*}{$\frac{\text { PRP group }(\mathrm{n}=20)}{\text { Mean } \pm \text { SD }}$} & \multirow{2}{*}{$\frac{\text { Control group }(\mathrm{n}=20)}{\text { Mean } \pm \text { SD }}$} & \multirow[b]{2}{*}{$p^{*}$} & \multirow[b]{2}{*}{ Post-power } \\
\hline & & & & \\
\hline \multicolumn{5}{|l|}{ Active } \\
\hline Baseline & $30.0 \pm 17.1^{\mathrm{a}}$ & $35.0 \pm 15.7^{\mathrm{a}}$ & 0.343 & 0.155 \\
\hline 2 weeks & $53.0 \pm 24.1^{\mathrm{b}}$ & $56.7 \pm 19.2^{\mathrm{b}}$ & 0.590 & 0.083 \\
\hline 6 weeks & $59.7 \pm 18.4 \mathrm{~b}^{\mathrm{c}}$ & $62.0 \pm 17.5^{\mathrm{b}}$ & 0.695 & 0.067 \\
\hline 12 weeks & $73.7 \pm 11.3^{c}$ & $67.5 \pm 19.2^{\mathrm{b}}$ & 0.218 & 0.231 \\
\hline Difference (2 weeks-Baseline) & $23.3 \pm 24.4$ & $21.8 \pm 20.7$ & 0.862 & 0.053 \\
\hline Difference (6 weeks-Baseline) & $29.8 \pm 19.0$ & $27.0 \pm 19.0$ & 0.650 & 0.073 \\
\hline Difference (12 weeks-Baseline) & $43.8 \pm 19.4$ & $32.5 \pm 20.9$ & 0.086 & 0.405 \\
\hline$p^{* *}$ & $<0.001$ & $<0.001$ & & \\
\hline Post-power & 1.000 & 1.000 & & \\
\hline \multicolumn{5}{|l|}{ Passive } \\
\hline Baseline & $33.0 \pm 16.7^{\mathrm{a}}$ & $37.5 \pm 16.3^{\mathrm{a}}$ & 0.395 & 0.134 \\
\hline 2 weeks & $58.2 \pm 22.9^{\mathrm{b}}$ & $60.5 \pm 17.3^{\mathrm{b}}$ & 0.728 & 0.064 \\
\hline 6 weeks & $62.5 \pm 17.0^{\mathrm{b}}$ & $65.2 \pm 15.7^{\mathrm{b}}$ & 0.600 & 0.081 \\
\hline 12 weeks & $77.0 \pm 9.7^{\mathrm{b}}$ & $69.7 \pm 17.2^{b}$ & 0.110 & 0.358 \\
\hline Difference (2 weeks-Baseline) & $25.3 \pm 22.9$ & $23.0 \pm 19.0$ & 0.737 & 0.063 \\
\hline Difference (6 weeks-Baseline) & $29.5 \pm 19.5$ & $27.8 \pm 17.9$ & 0.769 & 0.060 \\
\hline Difference (12 weeks-Baseline) & $44.0 \pm 22.7$ & $32.3 \pm 19.4$ & 0.087 & 0.403 \\
\hline$p^{* *}$ & $<0.001$ & $<0.001$ & & \\
\hline Post-power & 1.000 & 1.000 & & \\
\hline
\end{tabular}




\begin{tabular}{|c|c|c|c|c|}
\hline Changes from baseline $\mathrm{i}$ & $\begin{array}{l}\text { TABLE } 10 \\
\text { external rotation s }\end{array}$ & es between PRP anc & ontro & \\
\hline & PRP group $(n=20)$ & Control group $(n=20)$ & & \\
\hline Internal rotation & Mean \pm SD & Mean \pm SD & $p^{*}$ & Post-power \\
\hline Active & & & & \\
\hline Baseline & $41.7 \pm 13.5^{\mathrm{a}}$ & $46.7 \pm 13.7^{\mathrm{a}}$ & 0.256 & 0.203 \\
\hline 2 weeks & $62.7 \pm 16.0^{\mathrm{ab}}$ & $63.5 \pm 12.4^{\mathrm{b}}$ & 0.870 & 0.053 \\
\hline 6 weeks & $71.2 \pm 14.4^{\mathrm{bc}}$ & $66.5 \pm 13.5^{\mathrm{bc}}$ & 0.292 & 0.181 \\
\hline 12 weeks & $82.2 \pm 13.6^{c}$ & $71.7 \pm 17.3^{\mathrm{c}}$ & 0.040 & 0.546 \\
\hline Difference (2 weeks-Baseline) & $21.0 \pm 17.4$ & $16.8 \pm 12.4$ & 0.378 & 0.140 \\
\hline Difference (6 weeks-Baseline) & $29.5 \pm 17.5$ & $19.8 \pm 14.2$ & 0.060 & 0.472 \\
\hline Difference (12 weeks-Baseline) & $40.5 \pm 19.5$ & $25.0 \pm 15.5$ & 0.008 & 0.776 \\
\hline$p^{* *}$ & $<0.001$ & $<0.001$ & & \\
\hline Post-power & 1.000 & 1.000 & & \\
\hline Passive & & & & \\
\hline Baseline & $45.2 \pm 13.5^{\mathrm{a}}$ & $50.2 \pm 14.8^{\mathrm{a}}$ & 0.272 & 0.192 \\
\hline 2 weeks & $67.7 \pm 15.6^{\mathrm{b}}$ & $67.5 \pm 12.9^{\mathrm{b}}$ & 0.956 & 0.050 \\
\hline 6 weeks & $73.5 \pm 12.5^{\mathrm{bc}}$ & $71.2 \pm 13.3^{\mathrm{b}}$ & 0.587 & 0.083 \\
\hline 12 weeks & $83.7 \pm 12.4^{\mathrm{c}}$ & $75.7 \pm 15.5^{\mathrm{b}}$ & 0.081 & 0.416 \\
\hline Difference (2 weeks-Baseline) & $22.5 \pm 15.2$ & $17.3 \pm 12.4$ & 0.238 & 0.215 \\
\hline Difference (6 weeks-Baseline) & $28.3 \pm 15.9$ & $21.0 \pm 15.0$ & 0.147 & 0.304 \\
\hline Difference (12 weeks-Baseline) & $38.5 \pm 17.3$ & $25.5 \pm 16.5$ & 0.020 & 0.658 \\
\hline$p^{* *}$ & $<0.001$ & $<0.001$ & & \\
\hline Post-power & 1.000 & 1.000 & & \\
\hline
\end{tabular}

\section{DISCUSSION}

In the present study, we attempted to investigate the effectiveness of PRP therapy in patients with adhesive capsulitis. To the best of our knowledge, this study is the first in the literature to contribute to the improvement in shoulder ROM scores, particularly in active flexion, passive flexion, active abduction, passive abduction, and active external rotation in patients with adhesive capsulitis with the addition of intra-articular PRP to the treatment of patients who exercise regularly. Of note, our study results demonstrated that PRP therapy could not make an additional contribution to exercise therapy in the scales of VAS and SPADI in these patients. Immediately after treatment at two weeks, both groups showed a significant improvement in all scores of all scales. Moreover, this healing process continued dramatically until the end of the study. This effect reaffirms the importance of exercise in the adhesive capsule with the synergy of PRP on ROM.

Review of the literature reveals a limited number of data regarding PRP applications in adhesive capsulitis. The effectiveness of PRP treatment in the adhesive capsule was first shown as a case in $2015 .{ }^{[20]}$ In this case,
Aslani et al. ${ }^{[20]}$ applied PRP treatment seven months after the onset of symptoms. Stretching exercise was also recommended to the patient after each injection, and PRP was applied to the glenohumeral joint and the procedure was repeated after four weeks. Passive ROM by the goniometer, VAS, and Disability of Arm, Shoulder and Hand questionnaire was measured four weeks after the second application. Functional improvements of more than $70 \%$ based on the DASH questionnaire were observed. Moreover, healing outcomes were obtained in shoulder pain, flexion, abduction and external rotation. In our randomized-controlled study, the healing clinical results of the exercise and the combined PRP therapy were obtained, consistent with the aforementioned case.

Limitations in daily living activities due to pain and stiffness, impaired sleep quality due to nocturnal pain, fatigue, and depression can be seen in adhesive capsulitis. ${ }^{[21]}$ In conservative treatment, several non-operative treatment modalities have been identified, including non-steroidal anti-inflammatory drugs, physical therapy, hydrodilatation, intra-articular steroid injection, and intra-articular hyaluronic acid injection. ${ }^{[21]}$ Several complications such as fat atrophy, skin discoloration, 
weakness, and thinning in ligaments and tendons can occur following intra-articular steroid injection. ${ }^{[22,23]}$ If there is no response to these treatments, surgical treatment is indicated.

The exact cause of the lack of long-term effectiveness of intra-articular injection applications is still unclear, and alternative searches for the treatment are in progress. While the search for new treatments is ongoing, in a study by Barman et al., ${ }^{[24]}$ patients with adhesive capsulitis were divided into two groups and one group was given steroid and the other group PRP via intra-articular route. A total of 60 patients with adhesive capsulitis were assigned into two groups to receive $4 \mathrm{~mL}$ PRP and $4 \mathrm{~mL}$ corticosteroid via intra-articular injection. Both groups were given a routine home-based exercise program. The authors found that there were significant improvements in pain, disability, and ROM after 12 weeks in the PRP group. They also reported significant differences in all status of VAS and SPADI scores for all time points after treatment compared to baseline in both groups. In addition, these parameters were statistically significantly more improved over time in the PRP group compared to the corticosteroid group. On the other hand, Barman et al. ${ }^{[24]}$ found significant increases in all ROM scores in both groups. In the inter-group comparison, statistically significant improvements were shown in the internal rotation, external rotation, active abduction and passive abduction in the PRP group. In our study, we did not use corticosteroids and we described exercise patients as the control group. We observed a greater improvement in terms of ROM scores, compared to the control group, while the VAS and SPADI scores were similar to the control group. The discrepancy in the efficacy results between the two studies may be related to the dose and preparation of PRP. Also, patients having a secondary etiology (such as diabetes) leading to adhesive capsulitis were not included in this study.

In another study of Lin, ${ }^{[25]}$ the participants with adhesive capsulitis were divided into two groups as PRP and procaine, as a local anesthetic drug. The author found that the pain-point local injection with PRP was beneficial on the pain and the shoulder function, and the efficiency of PRP was also superior and longer than local procaine. To date, several studies have compared hyaluronic acid injection, steroid injection and physical therapy modality, and physical therapy modality was significantly superior to other modalities. ${ }^{[26,27]}$ In our study, the healing effects of effective exercise therapy on pain and disability were shown to reach a very dramatic high degree.
The PRP promotes pro-inflammatory mechanisms by the growth factor, cytokines, and bioactive proteins in alpha granules of platelets and decreases inflammation. It provides repair and regeneration of injured articular cartilage and tissue repair. ${ }^{[28,29]}$ In our study, positive results in joint movements obtained by adding PRP to exercise therapy may be associated with this mechanism.

Nonetheless, there are some limitations to this study. First, it could have been regulated with the placebo group receiving normal saline. However, we preferred applying exercise therapy studies with a longer follow-up period and including more patients as needed. Second, although the number of patients was sufficient to show the difference between time points in the groups, it was not enough to assess the difference between the groups according to the post-hoc power analysis. Nevertheless, while selecting patients with adhesive capsulitis, only patients with primary (idiopathic) etiology were included, and those with diabetes were, particularly, excluded. Indeed, this makes our study more valuable to observe the direct effectiveness of PRP.

In conclusion, home-based exercise therapy should be given more importance in patients with adhesive capsulitis. The addition of PRP to exercise treatment can improve patients' joint mobility, but not pain and disability in adhesive capsulitis. Further studies are needed to consider the effectiveness of PRP treatment in guidelines and to expand its administration in the clinical practice.

\section{Declaration of conflicting interests}

The authors declared no conflicts of interest with respect to the authorship and/or publication of this article.

\section{Funding}

The authors received no financial support for the research and/or authorship of this article.

\section{REFERENCES}

1. Bouaicha S, Wieser K, Kriechling P, Scholz-Odermatt SM. A large-scale assessment of the healthcare burden of adhesive capsulitis of the shoulder joint. Swiss Med Wkly 2020;150:w20188.

2. De Baets L, Vanbrabant S, Dierickx C, van der Straaten R, Timmermans A. Assessment of scapulothoracic, glenohumeral, and elbow motion in adhesive capsulitis by means of inertial sensor technology: A within-session, intra-operator and inter-operator reliability and agreement study. Sensors (Basel) 2020;20:876.

3. Koh KH. Corticosteroid injection for adhesive capsulitis in primary care: A systematic review of randomised clinical trials. Singapore Med J 2016;57:646-57. 
4. Bendich I, Rubenstein WJ, Cole BJ, Ma CB, Feeley BT, Lansdown DA. What is the appropriate price for platelet-rich plasma injections for knee osteoarthritis? A cost-effectiveness analysis based on evidence from level I randomized controlled trials. Arthroscopy 2020;36:1983-91.

5. Cardoso TB, Pizzari T, Kinsella R, Hope D, Cook JL. Current trends in tendinopathy management. Best Pract Res Clin Rheumatol 2019;33:122-40.

6. Angelo RL. Surgical management of proximal long head biceps tendon disorders. Sports Med Arthrosc Rev 2018;26:176-80.

7. Yerlikaya M, Talay Çaliş H, Tomruk Sütbeyaz S, Sayan H, Ibiş N, Koç A, et al. Comparison of effects of leukocyterich and leukocyte-poor platelet-rich plasma on pain and functionality in patients with lateral epicondylitis. Arch Rheumatol 2017;33:73-9.

8. Patel S, Dhillon MS, Aggarwal S, Marwaha N, Jain A. Treatment with platelet-rich plasma is more effective than placebo for knee osteoarthritis: A prospective, doubleblind, randomized trial. Am J Sports Med 2013;41:356-64.

9. de Jonge S, de Vos RJ, Weir A, van Schie HT, Bierma-Zeinstra SM, Verhaar JA, et al. One-year follow-up of platelet-rich plasma treatment in chronic Achilles tendinopathy: A double-blind randomized placebo-controlled trial. Am J Sports Med 2011;39:1623-9.

10. Cerza F, Carnì S, Carcangiu A, Di Vavo I, Schiavilla V, Pecora A, et al. Comparison between hyaluronic acid and platelet-rich plasma, intra-articular infiltration in the treatment of gonarthrosis. Am J Sports Med 2012;40:2822-7.

11. Filardo G, Kon E, Della Villa S, Vincentelli F, Fornasari PM, Marcacci M. Use of platelet-rich plasma for the treatment of refractory jumper's knee. Int Orthop 2010;34:909-15.

12. Yilmaz B, Kesikburun S. Plateletten zengin plazma uygulamaları. Türk Fiz Tip Rehab Derg 2013;59:338-44.

13. Barker SL, Bell SN, Connell D, Coghlan JA. Ultrasoundguided platelet-rich plasma injection for distal biceps tendinopathy. Shoulder Elbow 2015;7:110-4.

14. Barreto RB, Azevedo AR, Gois MC, Freire MRM, Silva DS, Cardoso JC. Platelet-Rich Plasma and Corticosteroid in the Treatment of Rotator Cuff Impingement Syndrome: Randomized Clinical Trial. Rev Bras Ortop (Sao Paulo) 2019;54:636-43.

15. Mazzocca AD, McCarthy MB, Chowaniec DM, Cote MP, Romeo AA, Bradley JP, et al. Platelet-rich plasma differs according to preparation method and human variability. J Bone Joint Surg [Am] 2012;94:308-16.

16. Roach KE, Budiman-Mak E, Songsiridej N, Lertratanakul Y. Development of a shoulder pain and disability index. Arthritis Care Res 1991;4:143-9.
17. Bumin G, Tuzun EH, Tonga E. The Shoulder Pain and Disability Index (SPADI): Cross-cultural adaptation, reliability, and validity of the Turkish version. Journal of Back and Musculoskeletal Rehabilitation 2008;21:57-62.

18. Noguchi K, Gel YR, Brunner E, Konietschke F. nparLD: An $\mathrm{R}$ software package for the nonparametric analysis of longitudinal data in factorial experiments. Journal of Statistical Software 2012;50:1-23.

19. Brunner E, Domhof S, Langer F, editors. Nonparametric analysis of longitudinal data in factorial experiments. New York: John Wiley \& Sons; 2002.

20. Aslani H, Nourbakhsh ST, Zafarani Z, Ahmadi-Bani M, Ananloo ME, Beigy M, et al. Platelet-rich plasma for frozen shoulder: A case report. Arch Bone Jt Surg 2016;4:90-3.

21. Le HV, Lee SJ, Nazarian A, Rodriguez EK. Adhesive capsulitis of the shoulder: Review of pathophysiology and current clinical treatments. Shoulder Elbow 2017;9:75-84

22. Xiao RC, Walley KC, DeAngelis JP, Ramappa AJ. Corticosteroid injections for adhesive capsulitis: A review. Clin J Sport Med 2017;27:308-20.

23. Song A, Higgins LD, Newman J, Jain NB. Glenohumeral corticosteroid injections in adhesive capsulitis: A systematic search and review. PM R 2014;6:1143-56.

24. Barman A, Mukherjee S, Sahoo J, Maiti R, Rao PB, Sinha $\mathrm{MK}$, et al. Single intra-articular platelet-rich plasma versus corticosteroid injections in the treatment of adhesive capsulitis of the shoulder: A cohort study. Am J Phys Med Rehabil 2019;98:549-57.

25. Lin J. Platelet-rich plasma injection in the treatment of frozen shoulder: A randomized controlled trial with 6-month follow-up. Int J Clin Pharmacol Ther 2018;56:366-71.

26. Hsieh LF, Hsu WC, Lin YJ, Chang HL, Chen CC, Huang V. Addition of intra-articular hyaluronate injection to physical therapy program produces no extra benefits in patients with adhesive capsulitis of the shoulder: a randomized controlled trial. Arch Phys Med Rehabil 2012;93:957-64.

27. Calis M, Demir H, Ulker S, Kirnap M, Duygulu F, Calis HT. Is intraarticular sodium hyaluronate injection an alternative treatment in patients with adhesive capsulitis? Rheumatol Int 2006;26:536-40.

28. Laver L, Marom N, Dnyanesh L, Mei-Dan O, EspregueiraMendes J, Gobbi A. PRP for degenerative cartilage disease: A systematic review of clinical studies. Cartilage 2017;8:341-64.

29. Lee HR, Park KM, Joung YK, Park KD, Do SH. Platelet-rich plasma loaded hydrogel scaffold enhances chondrogenic differentiation and maturation with up-regulation of $\mathrm{CB} 1$ and CB2. J Control Release 2012;159:332-7. 